\section{JTI}

JOURNAL OF

TRAUMA AND INJURY

Received: October 8, 2018

Revised: October 19, 2018

Accepted: October 22, 2018

\section{Correspondence to}

Hyung Jun Song, M.D.

Department of Emergency Medicine, Wonkwang University Sanbon Hospital, Wonkwang University School of Medicine, 321 Sanbon-ro, Gunpo 15865, Korea

Tel: $+82-31-390-2269$

Fax: +82-31-390-2348

E-mail: babydog7@hotmail.com

\title{
A Prospective, Randomized, Controlled Trial to Assess the Efficacy of a Multi-Dis- ciplinary Screening, Brief Intervention and Referral to Treatment Program for Patients with Fractures of the Oral and Maxillofa- cial Region Because of Alcohol-Related Injuries in the Emergency Department
}

\author{
Ja Heon Koo, M.D. ${ }^{1}$, Hyung Jun Song, M.D. ${ }^{1}$, Jun Hee Lee, M.D. ${ }^{1}$, \\ Jae Hyun Kim, M.D., Ph.D. ${ }^{2}$, Jung Woo Nam, D.D.S. ${ }^{3}$, Jae Eun Im, R.N. ${ }^{1}$ \\ Departments of ${ }^{1}$ Emergency Medicine, ${ }^{2}$ Neuropsychiatry, ${ }^{3}$ Oral and Maxillofacial Surgery, \\ Wonkwang University Sanbon Hospital, Wonkwang University School of Medicine, \\ Gunpo, Korea
}

Purpose: We have implemented a multi-disciplinary Screening, Brief Intervention and Referral to Treatment (SBIRT) protocol to prevent individuals who sustained alcohol-related traumatic injuries. We therefore conducted this single-center, prospective, randomized, controlled trial (RCT) to assess its efficacy.

Methods: All the enrolled patients $(n=30)$ were randomized to either the SBIRT group or the control group. In the current RCT, the proportion of the patients who reduced the amount of alcohol consumption and those who received a specialized treatment served as primary outcome measures. Moreover, changes in a 3-item version of the Alcohol Use Disorders Identification Test Consumption (AUDIT-C), Severity of Dependence Scale (SDS) and Kessler Psychological Distress Scale (K-6) scores at 3 months from baseline served as secondary outcome measures.

Results: At 3 months, the proportion of the patients who reduced the amount of alcohol consumption was significantly higher in the SBIRT group as compared with the control group ( $86.7 \%$ vs. $57.1 \%, p=0.02)$. Moreover, the proportion of the patients who received a specialized treatment was also significantly higher as compared with the control group $(26.7 \%$ vs. $1.4 \%, p=0.01)$. Furthermore, there were significant differences in changes in the AUDIT, SDS and K- 6 scores at 3 months from baseline between the two groups $(p<0.05)$.

Conclusions: In conclusion, our results indicate that the SBIRT is effective in reducing hazardous and harmful levels of drinking, the degree of alcohol dependence and that of psychological distress in at-risk drinkers.

Keywords: Alcohols; Alcohol drinking; Surgery, Oral; Interdisciplinary studies; SBIRT 


\section{INTRODUCTION}

The harmful alcohol use is a worldwide problem that results in millions of deaths. The annual number of deaths due to alcohol-related disease and injury in the US is estimated at approximately 100,000. Moreover, the socio-economic burden due to alcohol consumption is also estimated at more than USD 86 billion [1].

It is generally believed that alcohol is a contributing factor in $50 \%$ of all deaths from trauma; approximately $33-72 \%$ of patients sustaining a traumatic injury had alcohol detected from the blood. Of these, about 37-53\% had blood alcohol levels exceeding the legal limit [2]. The effects of alcohol consumption are characterized by increased confidence, impaired concentration, sensory and motor disturbances and reduced reflexes [3].

Alcohol intoxication (AI) is defined as a condition that results from alcohol ingestion to such an extent as to cause significant disturbances in consciousness, cognition, perception, behavior, functions or responses according to the international classification of diseases (ICD)-10 [4]. Its degree is increased with impaired consciousness progressing to coma with respiratory and cardiovascular depression. But its objective evaluation may be difficult. It is well established that $\mathrm{AI}$ is one of the risk factors of developing a variety of diseases including trauma. Thus, its association with a decreased capacity to recover from traumatic injury has been well described in the literature. Patients with AI are vulnerable to traffic accidents, nonfatal crushes or other types of accidents $[5,6]$.

In Korea, AI has been considered one of major problems; its prevalence is estimated at approximately $87.6 \%$ in Korean adults, thus being much higher as compared with other countries such as the US (64.9\%) or Canada (72.3\%). In addition, socio-economic loss due to alcohol-related problems is considerable and then annually increased in Korea [7].

As a serious public health problem, alcohol-related traumatic injury deserves special attention. Efforts have been therefore made to prevent the possible occurrence of alcohol-related trauma in individuals who are vulnerable to traumatic injuries from the AI; the Screening, Brief Intervention and Referral to Treatment (SBIRT) program has been widely used $[8,9]$.
Given the above background, we have implemented a multi-disciplinary SBIRT protocol to prevent individuals who sustained alcohol-related traumatic injuries. We therefore conducted this single-center, prospective, randomized, controlled trial (RCT) to assess its efficacy.

\section{METHODS}

\section{Study patients and setting}

The current single-center, prospective, RCT was conducted in a total of 36 patients who were admitted to department of oral and maxillofacial (OMF) surgery of our medical institution during a 3-month period ranging from February 1 to April 30, 2018. Inclusion criteria are as follows: 1) Men or women aged 16 years or older. 2) The patients visited the emergency department (ED) and then referred to the department of OMF surgery for operative or non-operative procedures. 3) The patients who sustained fractures of OMF region because of alcohol-related injuries. 4) The patients with a history of AI based on the ICD-10. And 5) the patients or their caregivers who were able to give informed consent. But we excluded the patients who were taking any treatments for alcohol-related problems at the time of study entry. We therefore enrolled a total of 30 patients $(n=30)$ in the current study; it was approved by the Institutional Review Board (IRB) of our medical institution (IRB No.: WMCSB201808-70). All the patients submitted a written informed consent for study participation. The current study was conducted in accordance with the Declaration of Helsinki.

\section{Rationale of sample size estimation}

For the current trial, we assumed that there are no significant differences in the patients' baseline findings. In addition, we conducted the current study to identify a significant difference in the degree of decrease in the amount of alcohol consumption by 0.50 standard deviation (SD). Considering a level of statistical significance of 0.05 , a statistical power of 0.80 and a drop-out rate of $20 \%$, we estimated the sample size per group at 15. 


\section{Evaluation tools}

\section{AUDIT}

The AUDIT is a proper estimate of hazardous and harmful levels of alcohol consumption [10]. Thus, it is designed to screen for excessive drinking and to assist in brief assessment. Currently, it is widely used ED and primary health care patients [11]. The AUDIT-C is a 3-item scale, and it predicts the alcohol-related morbidity [12]. It has a high specificity and sensitivity for weekly binge drinking (79-96\% and 5-83\%, respectively) $[13,14]$. Moreover, it has been reported that individuals with more than 4 drinks on a single occasion are at 2 -fold higher risks of sustaining an injury within the next 6 hours [15].

\section{Severity of dependence scale (SDS)}

SDS is a 5-item scale that evaluates the psychological aspects of dependence, such as impaired control over drug use, and its cross-cultural reliability and validity has been well described in the literature [16]. In addition, it is used to screen for the presence of substance-related disorder, for which a cut-off value was set at 3 [17].

\section{Kessler psychological distress scale (K-6)}

$\mathrm{K}-6$ is a 6-item scale that measures the emotional distress, and it is designed to assess psychological distress during the preceding month [18].

\section{A multi-disciplinary SBIRT protocol}

\section{Screening (S)}

The patients are evaluated with a baseline screening for the measurement of the harmful and hazardous alcohol consumption based on the amount and frequency of drinking using the timeline followback (TLFB).

\section{Brief intervention (BI)}

The patients receive a specialized brief intervention (BI) performed by a well-trained nurse. The BI is designed to complete a motivational interview for approximately 1520 minutes, thus attempting to deliver patient-oriented therapeutic approaches and thereby to promote and support positive changes in drinking behavior in patients with alcohol-related disorders.
The patients receive the $\mathrm{BI}$ while awaiting treatment, for which both the nurses and the patients sign a prescription for change. Then, details of the alcohol treatment and counseling are delivered to the patients; this is an important factor that encourages the patients to have an access to the treatment system.

Referral to treatment (RT)

The patients are referred to the department of OMF surgery for further evaluation and treatment.

\section{Patient evaluation and criteria}

For the current trial, we organized an SBIRT taskforce comprising an ED physician, an OMF surgeon, a psychiatrist and a nurse, each of whom was involved in his or her areas of expertise. At the ED, the patients submitted a written informed consent. The patients were evaluated using both standardized measures and semi-structured interviews, performed by a research nurse, with individuals and family members. At baseline, their baseline characteristics were analyzed through a questionnaire study. Data was collected by a well-trained researcher who was blinded to details of the patients. Then, they were also evaluated for high-risk drinking using the AUDIT-C, SDS, and K-6. At baseline, we evaluated at-risk drinkers as measured by the AUDIT-C $\geq 6[19,20]$.

In the current study, we used the TLFB to assess the alcohol consumption over a 14-day period. It is a method for retrospectively evaluating substance use based on recall of activities and events. Its applicability to alcohol consumption has been evaluated and it has provided precise information about the pattern and variability of alcohol consumption [21]. Then, we asked the patients about whether it was typical of their recent substance use. Thus, we recorded their typical pattern of alcohol consumption. This was followed by randomization of them into two groups: the SBIRT group and the control group. The patients of the SBIRT group received a multi-disciplinary SBIRT program.

The patients were followed up at 3 months using a telephone. Then, changes in outcome measures at 3 months from baseline were analyzed. This was followed by comparison of differences in such changes between the two groups. 
In the current study, the proportion of the patients who reduced the amount of alcohol consumption and those who received a specialized treatment served as primary outcome measures. Moreover, changes in the AUDIT-C, SDS and K-6 scores at 3 months from baseline served as secondary outcome measures. Furthermore, we also performed an intent-to-treat (ITT) analysis. The ITT set comprises all the randomized patients but those who failed to meet eligibility criteria at baseline, those who failed to receive the SBIRT although they were assigned to the SBIRT group and those who failed to efficacy analysis.

\section{Statistical analysis}

All data was expressed as mean \pm SD. Statistical analysis was done using the SPSS ver. 18.0 for windows (SPSS
Inc., Chicago, IL, USA). Differences in changes in efficacy outcome measures at 3 months from baseline between the two groups were analyzed using the Student's $t$-test. Moreover, we also analyzed the normality of data distribution. A $p$-value of $<0.05$ was considered statistically significant.

\section{RESULTS}

\section{Baseline characteristics of the patients}

A total of 30 patients were enrolled in the current study, who were equally assigned to two groups. But there was one case of lost-to-follow-up in the control group. Therefore, 15 patients of the SBIRT group and 14 of the control

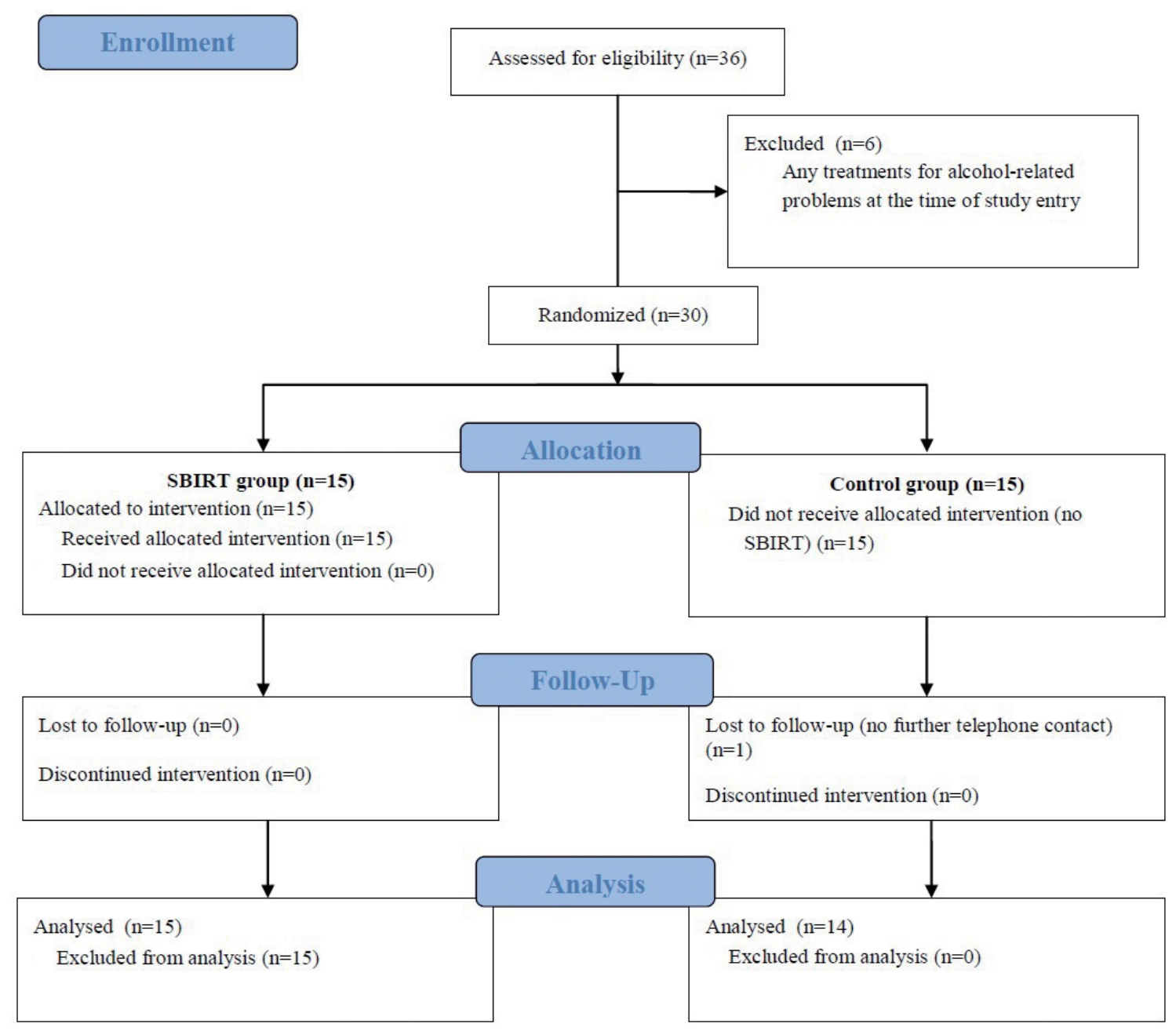

Fig. 1. Study flowchart. SBIRT: screening, brief intervention and referral to treatment. 
group completed the current study. The study flow chart was shown in Fig. 1.

The patients with alcohol-related facial fractures comprise 24 men and six women, with a mean age of $37.4 \pm 11.9$ years old. By causes of fractures, there were eight cases of assault, six cases of exercise or outdoor activities, three cases of fall and three cases of traffic accidents. Moreover, the fracture sites include mandible in 14 patients, maxilla in nine patients, zygoma in four patients, nasal-orbital-ethmoid complex in two patients and other

Table 1. Baseline characteristics of the patients

\begin{tabular}{|lc|}
\hline \multicolumn{1}{|c|}{ Value } \\
\hline Age (years) & $37.4 \pm 11.9$ \\
Men & $24(80.0)$ \\
Women & $6(20.0)$ \\
Causes of fractures & \\
Assault & $15(50.0)$ \\
Exercise of outdoor activities & $11(36.7)$ \\
Fall & $3(10.0)$ \\
Traffic accidents & $1(3.3)$ \\
Fracture sites & \\
Mandible & $14(46.7)$ \\
Maxilla & $9(30.0)$ \\
Zygoma & $4(13.3)$ \\
Nose-orbital-ethmoid complex & $2(6.7)$ \\
Other facial regions & $1(3.3)$ \\
Length of hospital stay (days) & $8.14 \pm 6.02$ \\
\hline
\end{tabular}

Values are presented as mean \pm standard deviation or number (\%). facial regions in one patient. Furthermore, the length of hospital stay was $8.14 \pm 6.02$ days. Baseline characteristics of the patients are represented in Tables 1 and 2; they were normally distributed and homogeneous.

\section{Outcomes of the SBIRT program}

At 3 months, the proportion of the patients who reduced the amount of alcohol consumption was significantly higher in the SBIRT group as compared with the control

Table 2. Baseline characteristics in each group

\begin{tabular}{|lcc|}
\hline & $\begin{array}{c}\text { SBIRT group } \\
(\mathbf{n}=15)\end{array}$ & $\begin{array}{c}\text { Control group } \\
(\mathbf{n}=14)\end{array}$ \\
\hline Age (years) & $41.0 \pm 8.7$ & $39.2 \pm 10.3$ \\
Sex & $12(80.0)$ & $11(78.6)$ \\
Men & $3(20.0)$ & $3(21.4)$ \\
Women & & \\
Causes of fractures & $8(53.3)$ & $7(50.0)$ \\
Assault & $5(33.3)$ & $6(42.9)$ \\
Exercise of outdoor activities & $1(6.7)$ & $1(7.1)$ \\
Fall & $1(6.7)$ & $0(0.0)$ \\
Traffic accidents & & \\
Fracture sites & $7(46.7)$ & $6(42.6)$ \\
Mandible & $5(30.0)$ & $4(28.6)$ \\
Maxilla & $2(13.3)$ & $2(14.3)$ \\
Zygoma & $0(0.0)$ & $2(14.3)$ \\
Nose-orbital-ethmoid complex & $1(6.7)$ & $0(0.0)$ \\
Other facial regions & $8.04 \pm 5.92$ & $8.09 \pm 5.97$ \\
Length of hospital stay (days) & & \\
\hline
\end{tabular}

Values are presented as mean \pm standard deviation or number (\%). SBIRT: screening, brief intervention and referral to treatment.

Table 3. Treatment outcomes

\begin{tabular}{|c|c|c|c|c|c|}
\hline & \multicolumn{4}{|c|}{ Measurement } & \multirow{3}{*}{$p$-value } \\
\hline & \multicolumn{2}{|c|}{ SBIRT group $(n=15)$} & \multicolumn{2}{|c|}{ Control group $(n=14)$} & \\
\hline & Baseline & 3 months & Baseline & 3 months & \\
\hline AUDIT-C & $11.8 \pm 5.7$ & $5.1 \pm 1.9$ & $11.8 \pm 4.5$ & $11.2 \pm 3.8$ & $0.02^{\mathrm{a}}$ \\
\hline SDS & $9.3 \pm 4.3$ & $2.4 \pm 1.2$ & $9.1 \pm 3.9$ & $8.8 \pm 1.4$ & $0.01^{\mathrm{a}}$ \\
\hline K-6 & $12.2 \pm 5.9$ & $6.2 \pm 2.2$ & $11.8 \pm 3.2$ & $10.3 \pm 3.5$ & $0.04^{\mathrm{a}}$ \\
\hline
\end{tabular}

Values are presented as mean \pm standard deviation.

SBIRT: screening, brief intervention and referral to treatment, AUDIT-C: alcohol use disorders identification test consumption, SDS: severity of dependence scale, K-6: Kessler distress scale.

aStatistical significance at $p<0.05$ by the Student's $t$-test. 
group ( $86.7 \%$ vs. $57.1 \%, p=0.02$ ). Moreover, the proportion of the patients who received a specialized treatment was also significantly higher as compared with the control group $(26.7 \%$ vs. $1.4 \%, p=0.01)$. Furthermore, there were significant differences in changes in the AUDIT, SDS and $\mathrm{K}-6$ scores at 3 months from baseline between the two groups $(p<0.05)$. The SBIRT outcomes at 3 months are represented in Table 3.

\section{DISCUSSION}

The SBIRT is designed to help those who are not strongly addicted to alcohol and who are still able to control over their amount and pattern of drinking even in the absence of professional help. Its implementation has been reported to effective in reducing harmful drinking habits and hospital re-admission [22-24]. This is also seen in our results; we found that the proportion of the patients who reduced the amount of alcohol consumption was significantly higher in the SBIRT group as compared with the control group. Moreover, our results also showed that the proportion of the patients who received a specialized treatment was also significantly higher as compared with the control group.

Previous studies have shown that the SBIRT was effective in improving the pattern of alcohol consumption in patients with hazardous and harmful drinking, alcohol dependence or psychological distress associated with alcohol use $[25,26]$. This is also seen in the current study; we found that there were significant differences in changes in the AUDIT-C, SDS, and K-6 scores at 3 months from baseline between the two groups. That is, the SBIRT was effective in reducing hazardous and harmful levels of drinking, the degree of alcohol dependence and that of psychological distress in at-risk drinkers. According to a systematic review of the published clinical trials, the SBIRT is a clinically beneficial modality in lowering risks of developing alcohol-related traumatic injuries. That is, Dinh-Zarr et al. [27] analysed the results of 17 clinical trials and reported that the SBIRT was effective in reducing the incidence of traumatic injuries by $27-65 \%$. But these authors added that cautions are needed in the interpretation of the results because of a lack of sufficiently large clinical studies [27]. According to Havard et al. [28], evaluating 11 RCTs, ED-based SBIRT was effective in halving odds of sustaining recurrent alcohol-related traumatic injuries although it failed to significantly reduce the amount of future alcohol consumption. Furthermore, Terrell et al. [29] analyzed analyzed the results of 19 RCTs and showed that the SBIRT was effective in the amount of weekly alcohol consumption in hazardous drinkers.

To summarize, our results are as follows: 1) At 3 months, the proportion of the patients who reduced the amount of alcohol consumption was significantly higher in the SBIRT group as compared with the control group ( $86.7 \%$ vs. $57.1 \%, p=0.02$ ). 2 ) At 3 months, the proportion of the patients who received a specialized treatment was also significantly higher as compared with the control group ( $26.7 \%$ vs. $1.4 \%, p=0.01$ ). And 3 ) there were significant differences in changes in the AUDIT, SDS and K-6 scores at 3 months from baseline between the two groups $(p<0.05)$.

Limitations of the current study are as follows: 1) We failed to measure blood alcohol concentrations; we analyzed AI based on a history of alcohol use. This might have caused a subjective bias. Blood alcohol level is commonly used to confirm the presence of AI. But this is not mandatory except for medico-legal cases such as traffic accident [30]. 2) We evaluated only the patients who were hospitalized at a single, secondary medical institution. We could not therefore completely rule out the possibility of selection bias. 3) We enrolled a small number of patients in the current study. 4) We evaluated the patients during a short follow-up period of 3 months. And 5) we performed a telephone interview rather than a face-to-face one at the outpatient clinic. This might have caused a bias in the assessment of treatment outcomes.

The ED plays a pivotal role in providing an important environment for identifying, intervening and connecting patients with treatment and recovery support, thus endeavoring to improve patient health and reduce healthcare utilization in patients who misuse alcohol. It therefore serves as the gateway to the acute health care system where there are consequences of the burden of at-risk alcohol use behavior.

Here, we propose that the SBIRT protocol be developed for the assessment and application of motivational inter- 
viewing techniques in the above patient population. Thus, the SBIRT program will have a positive effect in helping such patients receive a long-term rehabilitation. Sustainability and incorporation of the SBIRT program into the current ED practices deserve further efforts.

\section{CONCLUSION}

In conclusion, our results indicate that the SBIRT is effective in reducing hazardous and harmful levels of drinking, the degree of alcohol dependence and that of psychological distress in at-risk drinkers. But further large-scale, multi-center studies with a longer follow-up are warranted to establish our results.

\section{REFERENCES}

1. Willmore J, Marko TL, Taing D, Sampasa-Kanyinga H. The burden of alcohol-related morbidity and mortality in Ottawa, Canada. PLoS One 2017;12:e0185457.

2. Lange RT, Shewchuk JR, Rauscher A, Jarrett M, Heran MK, Brubacher JR, et al. A prospective study of the influence of acute alcohol intoxication versus chronic alcohol consumption on outcome following traumatic brain injury. Arch Clin Neuropsychol 2014;29:478-95.

3. Lange RT, Iverson GL, Franzen MD. Effects of day-of-injury alcohol intoxication on neuropsychological outcome in the acute recovery period following traumatic brain injury. Arch Clin Neuropsychol 2008;23:809-22.

4. Oscar-Berman M, Marinković K. Alcohol: effects on neurobehavioral functions and the brain. Neuropsychol Rev 2007;17: 239-57.

5. Waller PF, Hill EM, Maio RF, Blow FC. Alcohol effects on motor vehicle crash injury. Alcohol Clin Exp Res 2003;27:695-703.

6. Taylor B, Rehm J. The relationship between alcohol consumption and fatal motor vehicle injury: high risk at low alcohol levels. Alcohol Clin Exp Res 2012;36:1827-34.

7. Kim M. Analysis of the medical expense by alcohol attributed diseases. J Korean Alcohol Sci 2003;4:136-48.

8. Babor TF, McRee BG, Kassebaum PA, Grimaldi PL, Ahmed K, Bray J. Screening, brief intervention, and referral to treatment (SBIRT): toward a public health approach to the management of substance abuse. Subst Abus 2007;28:7-30.

9. Darnell D, Dunn C, Atkins D, Ingraham L, Zatzick D. A randomized evaluation of motivational interviewing training for mandated implementation of alcohol screening and brief intervention in trauma centers. J Subst Abuse Treat 2016;60:36-44.

10. Saunders JB, Aasland OG, Babor TF, de la Fuente JR, Grant M. Development of the alcohol use disorders identification test (AUDIT): WHO collaborative project on early detection of persons with harmful alcohol consumption--II. Addiction 1993;88:791-804.

11. Whitlock EP, Polen MR, Green CA, Orleans T, Klein J; U.S. Preventive Services Task Force. Behavioral counseling interventions in primary care to reduce risky/harmful alcohol use by adults: a summary of the evidence for the U.S. Preventive Services Task Force. Ann Intern Med 2004;140:557-68.

12. Proude EM, Britt H, Valenti L, Conigrave KM. The relationship between self-reported alcohol intake and the morbidities managed by GPs in Australia. BMC Fam Pract 2006;7:17.

13. Bradley KA, Bush KR, Epler AJ, Dobie DJ, Davis TM, Sporleder JL, et al. Two brief alcohol-screening tests from the alcohol use disorders identification test (AUDIT): validation in a female veterans affairs patient population. Arch Intern Med 2003;163:821-9.

14. Bush K, Kivlahan DR, McDonell MB, Fihn SD, Bradley KA. The AUDIT alcohol consumption questions (AUDIT-C): an effective brief screening test for problem drinking. Ambulatory care quality improvement project (ACQUIP). Alcohol use disorders identification test. Arch Intern Med 1998;158:1789-95.

15. Fillmore MT, Jude R. Defining "binge" drinking as five drinks per occasion or drinking to a .08\% BAC: which is more sensitive to risk? Am J Addict 2011;20:468-75.

16. Gossop M, Darke S, Griffiths P, Hando J, Powis B, Hall W, et al. The severity of dependence scale (SDS): psychometric properties of the SDS in English and Australian samples of heroin, cocaine and amphetamine users. Addiction 1995;90:607-14.

17. Lawrinson P, Copeland J, Gerber S, Gilmour S. Determining a cut-off on the severity of dependence scale (SDS) for alcohol dependence. Addict Behav 2007;32:1474-9.

18. Kessler RC, Andrews G, Colpe LJ, Hiripi E, Mroczek DK, Normand SL, et al. Short screening scales to monitor population prevalences and trends in non-specific psychological distress. Psychol Med 2002;32:959-76.

19. Dawson DA, Grant BF, Stinson FS, Zhou Y. Effectiveness of the derived alcohol use disorders identification test (AUDIT-C) in screening for alcohol use disorders and risk drinking in the US 
general population. Alcohol Clin Exp Res 2005;29:844-54.

20. DiGuiseppi C, Goss C, Xu S, Magid D, Graham A. Telephone screening for hazardous drinking among injured patients seen in acute care clinics: feasibility study. Alcohol Alcohol 2006;41:438-45.

21. Sobell LC, Agrawal S, Annis H, Ayala-Velazquez H, Echeverria L, Leo GI, et al. Cross-cultural evaluation of two drinking assessment instruments: alcohol timeline followback and inventory of drinking situations. Subst Use Misuse 2001;36:313-31.

22. Field CA, Caetano R, Harris TR, Frankowski R, Roudsari B. Ethnic differences in drinking outcomes following a brief alcohol intervention in the trauma care setting. Addiction 2010;105:6273.

23. Glass JE, Andréasson S, Bradley KA, Finn SW, Williams EC, Bakshi AS, et al. Rethinking alcohol interventions in health care: a thematic meeting of the International Network on Brief Interventions for Alcohol \& Other Drugs (INEBRIA). Addict Sci Clin Pract 2017;12:14.

24. Schermer CR, Moyers TB, Miller WR, Bloomfield LA. Trauma center brief interventions for alcohol disorders decrease subsequent driving under the influence arrests. J Trauma 2006;60:2934.
25. Field CA, Cochran G, Caetano R. Ethnic differences in the effect of drug use and drug dependence on brief motivational interventions targeting alcohol use. Drug Alcohol Depend 2012;126:21-6.

26. Montag AC, Brodine SK, Alcaraz JE, Clapp JD, Allison MA, Calac DJ, et al. Effect of depression on risky drinking and response to a screening, brief intervention, and referral to treatment intervention. Am J Public Health 2015;105:1572-6.

27. Dinh-Zarr T, Goss C, Heitman E, Roberts I, DiGuiseppi C. Interventions for preventing injuries in problem drinkers. Cochrane Database Syst Rev 2004;(30):CD001857.

28. Havard A, Shakeshaft A, Sanson-Fisher R. Systematic review and meta-analyses of strategies targeting alcohol problems in emergency departments: interventions reduce alcohol-related injuries. Addiction 2008;103:368-76; discussion 377-8.

29. Terrell F, Zatzick DF, Jurkovich GJ, Rivara FP, Donovan DM, Dunn CW, et al. Nationwide survey of alcohol screening and brief intervention practices at US Level I trauma centers. J Am Coll Surg 2008;207:630-8.

30. Touquet R, Csipke E, Holloway P, Brown A, Patel T, Seddon AJ, et al. Resuscitation room blood alcohol concentrations: oneyear cohort study. Emerg Med J 2008;25:752-6. 